\title{
DESIGN OF NEW GENERATION OF SERIOUS GAMES FOR PATIENTS WITH MODERATE COGNITIVE IMPAIRMENT
}

\author{
Barbara Carillon ${ }^{1}$, Brune Dirian-Angeli ${ }^{1}$, Gabrièle Breda ${ }^{2}$, Samuel Benveniste ${ }^{3}$ \\ and François Deparis ${ }^{1}$ \\ ${ }^{1}$ ARPAVIE, Issy-Les-Moulineaux, France \\ ${ }^{2}$ ALTRAN Resarch Department, Vélizy-Villacoublay, France \\ ${ }^{3}$ CEN Stimco - Broca Living Lab, Hôpital Broca, Paris, France
}

\begin{abstract}
Among the new challenges created by the aging population, which continues to increase significantly, many researchers have taken an interest in designing new services for people suffering from Alzheimer's disease such as serious games. They have proposed a diverse selection of guidelines for designing cognitive serious games, considering cognitive complexity and motivation. Generally, existing work rely on virtual reality or video interfaces. However, most of these games are not simple and effective enough to suit the needs of people suffering from Alzheimer's disease.

In this paper, a set of specific guidelines for designing and implementing effective serious games targeting patients with Alzheimer's disease and different disabilities are proposed. These guidelines aim to design serious games offering natural interaction helping people to communicate with the ability to capture their attention and to improve their self-esteem by encouraging them and highlighting their preserved abilities.
\end{abstract}

\section{KEYWORDS}

Alzheimer's Disease, Robot-Assisted Therapy, Humanoid Robot, Cognitive Stimulation, Serious Games

\section{INTRODUCTION}

Among the new challenges created by the aging population, which continues to increase significantly, many researchers have taken an interest in designing new services for people suffering from Alzheimer's disease. Alzheimer's disease and related diseases are part of the dementia category. They are characterized by a gradual deterioration of cognitive abilities caused by brain damage, with repercussions on the emotional states and behavior of patients. Thus, the symptomatology is diverse and it evolves slowly and gradually. The services designed for this population aim to lessen the impact of their disease in order to increase their autonomy and to improve their safety and well-being (Chibani A. et al, 2015).

In this context, many research projects focus on designing serious games for people with Alzheimer's (Castilla D. et al., 2013, Tong T. et al. 2017, Kort H., and Huisman C. 2017). These studies show clearly how such serious games can be beneficial for persons with Alzheimer's and their caregivers. Eichhorn et al demonstrate, in (Eichhorn C. et al, 2018), that exposure to these games improved people's cognition even weeks after they played them, partly countering the typical cognitive decline associated with Alzheimer's disease. Three main types of serious games are considered in the literature: cognitive, physical and social/emotional (McCallum S. and Boletsis C. 2013).

For people with Alzheimer's, cognitive exercises are very important and serious games play a key role to generate a positive atmosphere. Many researchers have proposed a diverse selection of guidelines for designing cognitive serious games, considering cognitive complexity and motivation. Tong et al. address different types of cognitive decline with potential game concepts such as object recognition, shifting and inhibition (Tong T. et al. 2017). 
Generally, existing work rely on virtual reality or video interfaces. However, most of these games are not simple and effective enough to suit the needs of people suffering of Alzheimer's disease. Indeed, according to Pyae et al., the game context should be familiar and game interactions should be easy (Pyae A. et al. 2016). In this context, some studies introduced social robots as a game, such as the Zora robot. Although it is successful in promoting physical exercise (Kort H., and Huisman C. 2017), a first trial with this robot for use in cognitive games showed its limitations due to ineffective verbal human-robot interaction.

Designing robotic system-based cognitive games for patients with Alzheimer's disease requires sophisticated and novel approaches. In order to capture the attention of patients and foster positive interactions, these systems need to be endowed with advanced, robust natural language processing, contextual non-verbal interaction (body language) and better decision-making capabilities.

To address these challenges, this paper presents a set of specific guidelines for designing and implementing effective serious games targeting patients with Alzheimer's disease and different disabilities. The proposed guidelines are based on preliminary research with healthcare professionals from a nursing home, in the context of memory workshops mediated by a robotic interface. They focus on:

(i) Natural interaction helping people to communicate. To deal with that, a "human-friendly" robot, called NAO, is used;

(ii) Capturing attention;

(iii) Encouraging and congratulating users;

(iv) Providing hints through different statement and gestures

(v) Fostering group interactions

In this paper, section 2 address in details these guidelines. Section 3 presents the designed games/exercises, and discusses their benefit for use with Alzheimer's patients before concluding with ongoing work.

\section{SERIOUS GAMES USING SOCIAL ROBOTS}

\subsection{New Design of Serious Games}

\subsubsection{Humanoid Robot as a Mediation Tool}

In coherence with its humanoid aspect, the robot has a participative role defined in the programming of the exercises. By mediation tool, we mean that it has the function of facilitating the therapist-patient-mediation relationship; mediation being the cognitive exercises for the memory workshop.

During workshops, the robot has two roles, animation and self-esteem improvement:

- The animation consists of interacting and communicating directly with the person by giving specific instructions on the exercise to be performed;

- The self-esteem part consists in encouraging the person and highlighting his/her preserved abilities.

To deal with that, the NAO robot was selected for many reasons:

i. Adequacy to the target population: the therapeutic workshops for people with Alzheimer's disease or a moderate-stage related illness with moderate psycho-behavioral symptoms aim to offer them psychosocial interventions to promote the use of all the preserved abilities of the person and a well-being in everyday life. Because of its social aspects including the ability to communicate verbally with body expressions and the ability to keep eye contact with people during an interaction, NAO robot is a stimulating mediating object to initiate speaking and to promote exchanges among people whose language is still preserved. These social aspects of the NAO robot are the major elements taken into account in the choice of this robot.

ii. Adequacy for target use: As the therapists in our team intended to use the robot for memory exercises and physical exercises, a humanoid robot seemed like the right choice: it would be able to show the physical exercises accurately and it would be more coherent for it to participate in memory exercises than a talking animal or hybrid robot.

iii. Practicality: Although its price remains quite high for a nursing home, it is easily available and already used in many different settings in France, with a rather efficient customer service, which is critical to ensure that operations run smoothly during the study that will be conducted. 
iv. Ease of programming: NAO offers a wide variety of pre-programmed functions and tools that enable researchers to quickly build new applications and iterate on their designs. Thus, although it is not the most technically advanced hardware, it appeared to offer the best mix of performance and ease of use for the work conducted during this study.

This choice was also supported by previous experiments too: some studies show that the NAO robot is judged more positively than other social robots such as the PARO robot, and a dog. In (Valentí S. et al., 2015), a pilot study was conducted in two phases in nursing homes and day care centers, with 201 people with Alzheimer's disease or related diseases to compare the effects on behavior and cognitive abilities of patients of three types interventions: the NAO robot, the PARO robot, and a dog. Groups of patients involved in NAO robot-assisted therapy showed a decrease in apathy as well as a measured decrease in Mini Mental State Examination (MMSE) scores. A recent study was conducted in a geriatric unit with 9 people with cognitive impairment to evaluate the effects of using a NAO-based intervention in psychomotor sessions on commitment, emotional state, well-being of the person as well as the appreciation of this type of session compared to a conventional therapy session. The first results show better commitment, positive answers and a greater satisfaction of patients during sessions attended by the robot (Rouaix, N. et al., 2017).

\subsubsection{Natural Interaction}

Communication modalities have also been an essential element in designing games in order to facilitate interaction between robot and patients. To deal with that, some natural aspects are considered to design natural human-robot interaction in this work such as:

- Natural Language (NL): Using spoken natural language (NL) has received increasing attention in human-involved robotics research. NL facilitates the communication between users and games/robots and helps to create natural human-environment interaction (Baraglia et al. 2016).

- Body Language Expression: Beyond verbal communication, some associated gestures are designed to support the robot's utterances, in order to give more expressive messages, for example, affirmative and negative gestures, or a specific posture for when the robot is waiting for an answer from the patient.

\subsubsection{Encouraging and Congratulating Users}

Boosting patients' self-esteem is one of the important aspects that should be considered when designing serious games for this population. In this work, two such features are considered: encouragement and congratulation, which consist in highlighting the patient's preserved abilities through statements and gestures of encouragement (e.g. "I know you can find the answer") and congratulation (e.g. "You made it !").

\subsubsection{Hinting}

In order to create a failure-free experience for patient, game designers should consider a hinting feature.

Indeed, the robot participates as a mediator in strengthening the identity of the elderly, which is often undermined by the experience of old age. To this end, it is important that patients do not experience failures that would highlight their impairments. Thus, in case of a wrong answer, the robot gives clues successively to help the patient in finding the correct answer with the therapist's support.

\subsubsection{Fostering Group Interaction}

Many studies observed improvements in cognitive functions through social activities. A recent study from the National Social Life, Health, and Aging Project found that participation in "congregant," "diverse," or "friends" social networks was significantly associated with good mental health in later life, especially with avoidance of depressive symptoms and their potential to impair cognition (Nelson LA, et al. 2013). Moreover, emulation helps people to engage more fully in tasks. This justifies designing games for use by a group of patients instead of individual sessions.

\subsection{Cognitive Training Games}

The memory workshop program is based on three exercises frequently used by psychologists, some of which refer to the psychological assessment from the Mini Mental State Examination. 


\subsubsection{Temporal Orientation Exercises}

The first exercise concerns temporal orientation. It consists in searching the date of the day sequentially, such as, the day, the month and the year with the possibility of referring to clues to find the correct answer. This exercise is related to the alteration of episodic memory, i.e. the storage and retrieval of acquired experiences in a specific spatio-temporal context (Giffard B., et al., 2001). This is the memory of facts personally experienced. In the context of Alzheimer's disease, erasure advances in reverse order: patients forget recent facts first and progressively forget older facts, for example leading them to believe they live in the time of their childhood instead of present times. Patients forget the events that they experience, which generates a disorientation in time and space. Reorienting patients over time, at the beginning of the exercise, helps to limit the feeling of disorientation and anxiety.

\subsubsection{Semantic Memory Exercises}

The second exercise focuses on semantic memory. Semantic memory is the memory of concepts, ideas and facts that are independent of the spatio-temporal framework. It stores the learned general knowledge and it is queried when humans must give the spelling or the meaning of a word, or to remember a general fact such as the name of the president, capital city of a country, etc. (Giffard B., et al., 2001).

The proposed exercise consists in giving a random letter of the alphabet and asking patients to propose a list of categorized words, such as, first names, verbs or common names, starting with the given letter. Proposing cognitive exercises with the ability to access to semantic memory has many interests such as maintaining this form of memory while promoting a situation of success for the patient.

\subsubsection{Short and Long Term Memory Training}

The third exercise focuses on working memory. This latter is a short-term memory and is the most requested. It allows (i) to temporarily store data, such as a phone number, a path, etc.; (ii) to carry out mental operations such as calculations; or (iii) to follow reasoning at several stages, for example, applying instructions and following a path (Giffard B., et al., 2001). The alteration of this memory makes patients' attentional resources difficult to mobilize and very labile. The evolution of cognitive neuropsychology has allowed the development of specific and targeted methods of deficit brain function. These new approaches are used especially for verbal working memory. Propose exercises for specific cognitive rehabilitation to this form of memory had been efficiency shown in (Seron X., et al., 2008).

As this memory has a moderate alteration for the monitored population, the goal is to retrain it during a therapeutic workshop. In fact, the proposed exercise consists in listening to four words from the same lexical category, and recalling them after some delay. People gradually learn the words in a repetition task to generate memory encoding. A delayed recall is performed after an interfering task based on mental calculation such as reciting a multiplication table. This exercise also refers to several Mini Mental State Examination items.

\section{CONCLUSION}

This paper proposes a set of specific guidelines for designing and implementing effective serious games targeting patients with Alzheimer's disease and different disabilities. These guidelines focus on providing natural interaction helping people to communicate with the ability to capture their attention and to improve their self-esteem. The proposed approach is based on introducing a mediator tool such as the humanoid NAO robot. Indeed, the design of this robot has a soothing, attractive and playful effect. Furthermore, introducing a mediator tool is able to improve the interaction and the communication between the therapist and patients with behavioral disorders. It is important that this approach is not intended to suit all kinds of patients: dementia is a progressive disease, and the use of NAO is, in our view, most suited for people with moderate cognitive impairment. For example, animal robots with fewer interactional modalities such as PARO, which are less demanding for participants, seem to be more suited for interventions targeting people with more advanced dementia. A promising use for such robots is pain relief during medical care (Demange et al., 2019), for which NAO would be rather impractical to use. Indeed, the gradual deterioration of language, reasoning and comprehension abilities during the illness need an adaptation regarding to communication 
modality during the interaction. Focusing, at an advanced stage, on nonverbal communication and sensory stimulation in the relation favors a valorization of the preserved capacities and avoids any form of defeat. In comparison with the NAO robot, the choice of a robot such as PARO at this stage seems more relevant and adapted.

The ongoing works focus on the implementation of the proposed guidelines according to a robot cognitive architecture, introduced in previous work (Ayari et al. 2017), with an emphasis on system aspects according to multi-agents and service-oriented computing paradigms, such as, the natural language understanding model, reasoning mechanisms, and communication capabilities.

\section{REFERENCES}

Chibani A. et al. 2015. Using cognitive ubiquitous robots for assisting dependent people in smart spaces. In Intelligent Assistive Robots. Springer.

Castilla D. et al., 2013. Process of design and usability evaluation of a telepsychology web and virtual reality system for the elderly: Butler, International Journal of Human-Computer Studies, Vol. 71, No. 3, pp 350-362.

Demange, M., et al., 2019. Development of a robot-assisted intervention for the management of acute pain situations in dementia: framework and results from a feasibility study. Journal of pain research (in press).

Giffard B., et al., 2001. Le vieillissement de la mémoire: vieillissement normal et pathologique. Gérontologie et société, Vol. 24, No. 2, pp 44-45.

Kort H., and Huisman C. 2017; Care Robot ZORA in Dutch Nursing Homes; An Evaluation Study. Studies in health technology and informatics, 242, 527-534.

McCallum S. and Boletsis C. 2013. A taxonomy of serious games for dementia. In Games for Health, Springer Vieweg, Wiesbaden, pp. 219-232

Nelson LA, et al. 2013. Social engagement and physical and cognitive health among American Indian participants in the health and retirement study. J Cross Cult Gerontol, VOL. 28, No. 4, pp 453-456.

Pyae A. et al. 2016, When Japanese elderly people play a Finnish physical exercise game: a usability study. J. Usability Stud. Vol. 11, No. 4, pp 131-152.

Rouaix, N. et al. 2017. Affective and engagement issues in the conception and assessment of a Robot-Assisted psychomotor therapy for persons with dementia. Frontiers in psychology, 8, pp 950.

Seron, X., Rossetti, Y., Vallat-Azouvi, C., Pradat-Diehl, P., \& Azouvi, P. (2008). La rééducation cognitive. Revue neurologique, 164, S154-S163.

Valentí S. et al. 2015. Social robots in advanced dementia. Frontiers in aging neuroscience, 7, pp. 133.

Ayari, N., et al. 2017. Contextual Knowledge Representation and Reasoning Models for Autonomous Robots. In AAAI Fall Symposium Series, Washington USA.

Baraglia J. et al. 2016, Initiative in robot assistance during collaborative task execution, In 11th ACM/IEEE International Conference on Human-Robot Interaction (HRI), 67-74.

Bouchard B. et al, 2012. Developing serious games specifically adapted to people suffering from Alzheimer. In International Conference on Serious Games Development and Applications, Springer, Berlin, Heidelberg, pp. 243-254.

Chebaa B. et al. 2016. Multimodal analysis of serious games for cognitive and physiological assessment. In Proceedings of the 9th ACM International Conference on PErvasive Technologies Related to Assistive Environments, p. 30.

Eichhorn C. et al, 2018. Innovative Game Concepts for Alzheimer Patients. In International Conference on Human Aspects of IT for the Aged Population, Springer, Cham, pp. 526-545

Tong T. et al. 2017. Serious games for dementia. In Proceedings of the 26th International Conference on World Wide Web Companion, Perth, Australia, pp. 1111-111 